\title{
A negative D-dimer result and low risk clinical status effectively ruled out DVT in symptomatic patients
}

\author{
Aschwanden $M$, Labs KH, Jeanneret $C$, et al. The value of rapid D-dimer testing combined with structured clinical \\ evaluation for the diagnosis of deep vein thrombosis.J Vasc Surg 1999 Nov;30:929-35.

\begin{abstract}
QUESTION: Can a D-dimer assay, alone or combined with structured clinical risk assessment, be used to rule out deep venous thrombosis (DVT) in symptomatic patients?
\end{abstract}

Design

A blinded comparison of D-dimer test results plus results from a structured clinical assessment with duplex ultrasonographic scanning (DUS).

Setting

A university hospital in Basel, Switzerland.

\section{Participants}

Source of funding: none stated.

For correspondence: Dr KA Jaeger,

Department of

Angiology, University

Hospital, University of

Basel, Petersgraben 4,

CH-4031 Basel,

Switzerland. Fax +41

612655356

360 consecutive patients were screened, and 343 participants, who had complete information (median age $61 \mathrm{y}$, age range 17 to $94 \mathrm{y}, 61 \%$ women, 52\% inpatients, 398 limbs), were investigated for suspected DVT. No exclusion criteria were specified.

Description of tests and diagnostic standard A D-dimer analysis was done (SimpliRED, Agen Biomedical, Brisbane, Australia) initially. Patients were then divided into groups at low or high risk for DVT based on a previously validated clinical risk assessment

D-dimer assay and clinical assessment to diagnose deep venous thrombosis (DVT) in symptomatic limbst

\begin{tabular}{|c|c|c|c|c|c|}
\hline Test strategy & DVT & Sensitivity & Specificity & $+\mathrm{LR}$ & $-\mathrm{LR}$ \\
\hline \multirow[t]{3}{*}{ D-dimer alone } & All & $87 \%$ & $57 \%$ & 2.0 & 0.3 \\
\hline & Proximal & $89 \%$ & $55 \%$ & 2.0 & 0.02 \\
\hline & Calf & $81 \%$ & $50 \%$ & 2.0 & 0.4 \\
\hline \multirow[t]{3}{*}{ D-dimer plus clinical assessment } & All & $96 \%$ & $46 \%$ & 1.8 & 0.09 \\
\hline & Proximal & $98 \%$ & $43 \%$ & 1.7 & 0.05 \\
\hline & Calf & $91 \%$ & $38 \%$ & 1.5 & 0.2 \\
\hline
\end{tabular}

tLRs defined in glossary and calculated from data in article. score* calculated by using data on the presence of cancer, immobilisation, recent surgery, localised tenderness, swelling, pitting edema, and collateral superficial veins. Data were compiled by an examiner who was blinded to the D-dimer test results. DUS (diagnostic standard) was done in a blinded fashion to identify all (proximal and calf) DVT. All scans were conclusive.

\section{Main outcome measures}

Sensitivity and specificity for the diagnosis of DVT for patients and limbs using D-dimer test results alone and combined with a clinical risk assessment.

\section{Main results}

Of the 343 patients, $71 \%$ were at low risk for DVT. 16\% had proximal DVT, and 5\% had isolated calf DVT. The sensitivity of D-dimer assay was $89 \%$ in detecting proximal DVT and $87 \%$ for all DVT; when combined with a clinical assessment, sensitivity was $98 \%$ for proximal DVT and 96\% for all DVT (table).

\section{Conclusion}

A screening procedure based on negative D-dimer assay results combined with low risk clinical status effectively ruled out deep venous thrombosis (high sensitivity) in symptomatic patients.

* Wells PS, Anderson DR, Bormanis J, et al. Lancet 1997;350:1795-8.

\section{COMMENTARY}

In patients who present with a first episode of suspected DVT, DUS of the proximal (thigh) veins is usually done as a screening test. In patients with an abnormal scan, proximal DVT is confirmed, and in those with a normal scan, DUS is repeated within 7 days to exclude a distal (calf) DVT that may extend proximally. Recent studies have established that the combination of a negative D-dimer test result with a normal DUS or normal impedance plethysmographic measurement obviates the need for repeated non-invasive testing. . $^{-3}$

Aschwanden and Lennox and their associates attempt to further simplify the diagnostic assessment of suspected DVT by investigating whether a negative D-dimer test result combined with a low clinical likelihood for DVT obviates the need for DUS. This research is clinically important because approximately $50 \%$ of patients with suspected DVT have a low clinical likelihood of DVT. ${ }^{2}{ }^{3}$ Thus, the potential exists for substantial cost savings. In addition, because DUS may not be available on weekends or evenings, many patients with suspected DVT will receive empiric anticoagulant therapy unnecessarily until DUS is done, unless it is possible to rule out DVT with venography.

The D-dimer test investigated in these studies is a whole blood agglutination assay that is specific for degradation products of thrombus specific, cross linked fibrin. A result, based on a subjective assessment, is obtained within 5 minutes. As with other D-dimer tests, the SimpliRED assay is used as a "rule out" test, with a negative result used to exclude DVT. A positive result is non-specific and can be caused by DVT or such other conditions as soft tissue injury, infection, haematoma, malignancy, or pregnancy, all of which can result in elevated plasma D-dimer levels.

These studies suggest that in patients with suspected DVT, the combination of a negative D-dimer test result and a low clinical likelihood reliably excludes

DVT, thereby obviating the need for DUS. Both studies were well designed, blinded, and compared a D-dimer test plus a structured clinical assessment with a diagnostic reference standard, DUS. These studies differ from other diagnostic studies because both inpatients and outpatients were assessed, thereby increasing the generalisability of the results. Furthermore, DUS, not venography, was used as the diagnostic standard to assess proximal and calf DVT. DUS is essentially as accurate as venography for the diagnosis of symptomatic proximal DVT, but DUS for calf DVT has not been adequately evaluated and may continued on next page 